RESEARCH PAPER RP639

Part of Bureau of Standards Journal of Research, Vol. 12, February 1934

\title{
A METHOD OF ADJUSTING THE TEMPERATURE COEFFI- CIENT AND RESISTANCE OF LOW-VALUED RESISTANCE STANDARDS
}

\author{
By Frank Wenner and James L. Thomas
}

\section{ABSTRACT}

It is shown (1) that the temperature coefficient of low-valued resistance standards, which are of the 4-terminal type, may be either larger or smaller than the temperature coefficient of the resistance material used in their construction; (2) that in many cases it is possible to adjust the temperature coefficient to a practically zero value at a selected temperature; and (3) that the resistance may be decreased without the addition of a shunt, and without disturbing brazed connections between the resistance material and the copper terminals.

\section{CONTENTS}

I. Introduction

II. Theory

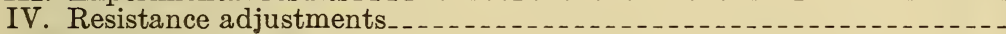

V. Load coefficients

\section{INTRODUCTION}

Low-valued resistance standards not infrequently have temperature coefficients differing considerably from that of the resistance material used in their construction. Such standards are of the 4-terminal type and the resistance element usually consists of two or more manganin wires in parallel or one or more manganin sheets in parallel. These wires or sheets are generally brazed to copper terminal posts and, since the copper has a relatively high temperature coefficient, it has generally been considered advisable to attach the potential connections in such a manner as to include a minimum amount of copper resistance between them. In fact, in many cases the potential connections are made directly on the manganin.

It is the purpose of this paper to point out, in case of an unsymmetrical construction: (1) that even with the potential connections made directly on the manganin, the copper terminals may be a factor affecting the temperature coefficient of the resistance standard; (2) that with the potential connections on the manganin the temperature coefficient may be changed by changing the section of the copper current posts; (3) that with the potential connections made on the copper current posts the temperature coefficient of the resistance standard may be either greater or less than the temperature coefficient of the manganin; (4) that with the potential connections on the copper current posts, the temperature coefficient and resistance may 
be changed by changing the position of the potential connections or the section of the current posts, or both; (5) that there is a fairly definite relation between the change in temperature coefficient of a resistance standard, the temperature coefficient of the copper current posts and the change in resistance of the standard brought about by either a change of the section of the current posts or movement of the potential connections to other points on the current posts. More specifically, when the temperature coefficient is changed in this way, the magnitude and the direction of the change may be calculated from the temperature coefficient of the current posts and the observed change of the resistance; (6) that lowering the points of attachment of the potential connections on the current posts or a reduction of the sections of the current posts below the upper manganin wire or upper

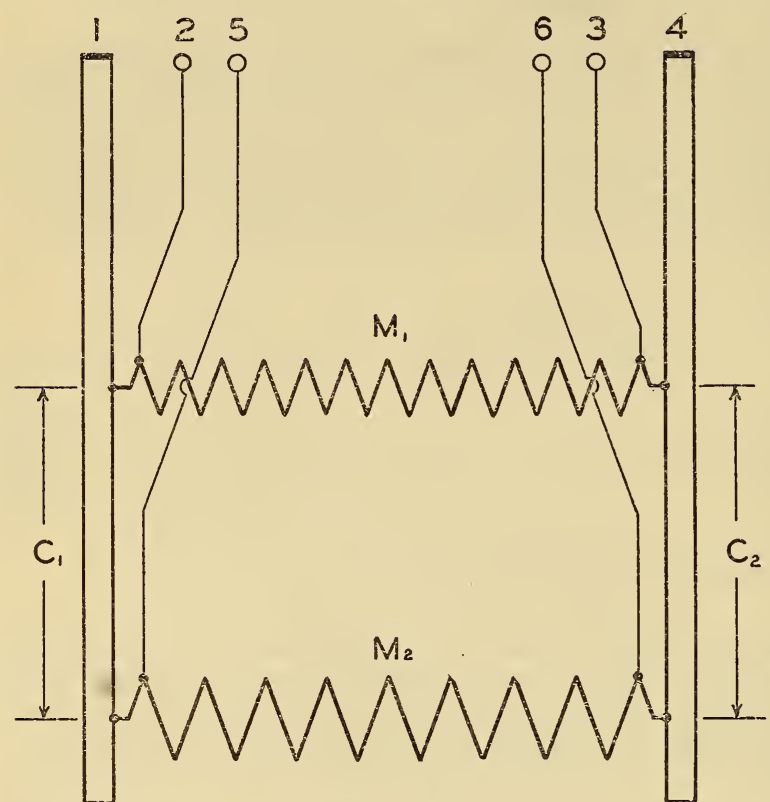

FIGURE 1.-Resistance standard with two sets of potential terminals.

Here 1 and 4 are current terminals, 2 and 3 are one and 5 and 6 are another set of potential terminals, $M_{1}$ and $M_{2}$ are the resistances of two manganin wires connected in parallel, and $C_{1}$ and $C_{2}$ are the resistances of the parts of the copper current posts between the manganin wires. Since the temperature coefficient of $C_{1}$ and $C_{2}$ is high relative to that of $M_{1}$ and $M_{2}$ a change in temperature causes a change in the current distribution between $M_{1}$ and $M_{2}$. Therefore not only the resistance but also the temperature coefficient depends on which set of potential terminals is used

valued 4-terminal resistance standards even edge of the manganin sheet or sheets and above the points of attachment of the potential connections lowers the resistance and the temperature coefficient while a reduction of the section of the resistance material increases the resistance without materially changing the temperature coefficient; and (7) that changing the temperature coefficient by changing points of attachment of the potential connections or sections of current posts has no material effect on the load coefficient.

\section{THEORY}

That the copper terminals may affect the temperature coefficient of lowwhen the potential terminals are connected directly to the manganin may be seen by considering the arrangement shown in figure 1. The essential parts are 2 manganin wires, shown as resistances; 2 current posts, and 4 potential leads. Here 1 and 4 are current terminals, 2 and 3 are one set of potential terminals, and 5 and 6 are another set of potential terminals, giving in effect two 4-terminal resistances which will be designated $R_{1234}$ and $R_{1564}$. Let $M_{1}$ be the resistance of the upper and $M_{2}$ be the resistance of the lower branch, each considered for the 
present as made up entirely of manganin, and $C_{1}$ and $C_{2}$ be the resistances of the parts of the current posts between the manganin wires. Then if $I$ is the current entering through terminal 1 and leaving through terminal $4, I_{1}$ is the current in the upper and $I_{2}$ is the current in the lower manganin wire, it is easily seen that

and

$$
\frac{I_{1}}{I}=\frac{M_{2}+C_{1}+C_{2}}{M_{1}+M_{2}+C_{1}+C_{2}}
$$

$$
\frac{I_{2}}{I}=\frac{M_{1}}{M_{1}+M_{2}+C_{1}+C_{2}}
$$

Since the proportional change of $C_{1}$ and $C_{2}$ with temperature is large in comparison with that of the manganin, if $M_{1}$ and $M_{2}$ change the same proportional amount for the same change in temperature, $\frac{I_{1}}{I}$ increases with an increase of the temperature and $\frac{I_{2}}{I}$ decreases with an increase of the temperature. Consequently, if 2 and 3 are used as potential terminals, the proportional increase of the ratio of the potential drop to the current $I$, which is the 4-terminal resistance $R_{1234}$, is greater than the proportional increase of $M_{1}$ or $M_{2}$. Likewise, if 5 and 6 are used as potential terminals, the proportional increase of the 4 -terminal resistance $R_{1564}$, with temperature, is less than the proportional increase of $M_{1}$ or $M_{2}$. Incidentally whether or not the individual temperature coefficients of $M_{1}$ and $M_{2}$ are equal, the rate of increase of $R_{1234}$, with temperature, will be greater than that of $R_{1564}$. Obviously, if the section of either current post between the upper and lower wires were reduced, this would increase the difference in the rate of change of $R_{1234}$ and $R_{1564}$, with temperature.

If the potential connections 2 and 3 are made on the copper current posts at points opposite to the points of attachment of the upper manganin wire, the resistance

$$
R_{1234}=\frac{M_{1}\left(M_{2}+C_{1}+C_{2}\right)}{M_{1}+M_{2}+C_{1}+C_{2}}
$$

If the potential connections 5 and 6 are made on the copper current posts at points opposite the point of attachment of the lower manganin wire, the resistance

$$
R_{1564}=\frac{M_{2} M_{1}}{M_{1}+M_{2}+C_{1}+C_{2}}
$$

That there is of necessity some brazing material-and usually some copper resistance-in each branch follows from the fact that the threeway electrical junctions between the wires and upper and lower ends of the current posts cannot be considered as points but must be considered as surfaces. These surfaces may or may not intersect boundaries between the manganin and brazing material, but never quite coincide with them nor in effect lie entirely within the manganin. 
Each branch, therefore, contains some brazing material and usually some copper but not necessarily all of a manganin wire. Let $D_{1}$ be the contribution of the brazing material and copper, and $M_{1}^{\prime}$ be limited to the contribution of the manganin to the resistance of the upper branch. Also let $D_{2}$ and $M_{2}{ }^{\prime}$ apply in like manner to the lower branch. Further let $D \equiv\left(D_{1}+D_{2}\right) / 2, M \equiv\left(M_{1}{ }^{\prime}+M_{2}{ }^{\prime}\right) / 2$ and $C \equiv\left(C_{1}+\right.$ $\left.C_{2}\right) / 2$. If then $M_{1}{ }^{\prime}$ and $M_{2}{ }^{\prime}$ are nearly equal, and both $C$ and $D$ are small in comparison with $M$, neglecting second and higher order terms, it follows that the resistance

$$
R_{1234}=\frac{M}{2}+\frac{D}{2}+\frac{C}{2}
$$

and the resistance

$$
R_{1564}=\frac{M}{2}+\frac{D}{2}-\frac{C}{2}
$$

Similar approximate solutions for $n$ wires of nearly equal resistance uniformly spaced along the current posts between points of attachment of the potential connections, are

$$
R_{1234}=\frac{M}{n}+\frac{D}{n}+\frac{(2 n-1) C}{3 n}
$$

and

$$
R_{1564}=\frac{M}{n}+\frac{D}{n}-\frac{(n+1) C}{3 n}
$$

If $M, C$, and $D$ apply at a particular temperature, $25 \mathrm{C}$ for example at some other temperature $t$

$$
\begin{aligned}
R_{1234}=\frac{M}{n}\left[1+\alpha(t-25)+\beta(t-25)^{2}\right]+\frac{D}{n}[ & +\delta(t-25)] \\
& +\frac{(2 n-1) C}{3 n}[1+\gamma(t-25)]
\end{aligned}
$$

and

$$
\begin{aligned}
R_{1564}=\frac{M}{n}\left[1+\alpha(t-25)+\beta(t-25)^{2}\right]+\frac{D}{n} & {[1+\delta(t-25)] } \\
& -\frac{(n+1) C}{3 n}[1+\gamma(t-25)]
\end{aligned}
$$

Here $\alpha$ and $\beta$ are the averages of the temperature coefficients of the manganin wires, $\gamma$ is the temperature coefficient of the copper current posts, and $\delta$ is the weighted average of the temperature coefficients of the brazing material and copper resistances in the branches, all applying with a reference temperature of $25 \mathrm{C}$. If additional second and higher order terms are neglected

$$
\begin{aligned}
R_{1234}=N\left[1+\frac{D}{n N}+\frac{(2 n-1) C}{3 n N}\right. & +\alpha(t-25)+\beta(t-25)^{2} \\
& \left.+\frac{D}{n N} \delta(t-25)+\frac{2 n-1}{3 n N} C \gamma(t-25)\right]
\end{aligned}
$$

and 


$$
\begin{aligned}
R_{1564}=N\left[1+\frac{D}{n N}-\frac{(n+1) C}{3 n}+\right. & \alpha(t-25)+\beta(t-25)^{2} \\
& \left.+\frac{D}{n N} \delta(t-25)-\frac{n+1}{3 n N} C \gamma(t-25)\right]
\end{aligned}
$$

where $N$ is the average resistance of the wires divided by the number. It will be seen that the temperature coefficient of $R_{1234}$ is larger than the temperature coefficient of $R_{1564}$ by $\gamma C / N$, that is

$$
\Delta R_{1234} / N \Delta t-\Delta R_{1564} / N \Delta t=\gamma C / N
$$

also that at $25 \mathrm{C}$

$$
R_{1234} / N-R_{1564} / N=C / N
$$

consequently the difference of the temperature coefficients is equal to the temperature coefficient of the copper times the proportional difference of the resistances.

If the resistance element is in the form of a sheet or number of sheets in parallel with the upper edge on a level with the points of attachment of the potential connections 2 and 3 and the lower edge on a level with the points of attachment of the potential connections 5 and 6 , the arrangement would be somewhat similar to that which would be obtained with a large number of similar wires uniformly spaced.

Ordinarily $\alpha$ has a value in the range from -0.00001 to +0.00003 , $\beta$ has a value in the range from -0.0000002 to $-0.0000006, \gamma$ is approximately 0.0038 , if the current posts are of copper having the normal conductivity; $\delta$ is less than 0.0038 ; and in many, if not most, cases, $D \delta / n N$ is less than 0.00001 . If, therefore, $C / N$ is as large as 0.01 , it is very probable that at temperatures in the vicinity of $25 \mathrm{C}$, the resistance $R_{1234}$ will increase with an increase of the temperature and that the resistance $R_{1564}$ will decrease with an increase of the temperature. If the two sets of potential connections were removed and other potential connections made on the current posts at points between the upper and lower wires or upper and lower edges of the manganin sheet, the resistance would be less than $R_{1234}$ and greater than $R_{1564}$. Also, the rate of change of the resistance with temperature would be less than that of $R_{1234}$ and greater than that of $R_{1564}$.

Consequently if $C / N$ is sufficiently large, by a proper selection of the point of attachment of the potential connections a zero rate of change of resistance with temperature at a selected temperature, such as 20 or $25 \mathrm{C}$, may be realized. Further, a reduction of the section of the current posts between the upper and lower manganin wires or upper and lower edges of the manganin sheet and above the points of attachment of the potential connection decreases the resistance and the rate of increase of the resistance with temperature. Also, a decrease of the section of the current posts between the upper and lower manganin wires or upper and lower edges of the manganin sheet and below the points of attachment of the potential connections increases the resistance and the rate of increase of the resistance with temperature. Finally, any relatively small change in resistance made by changes wholly on the current posts changes the rate of change of resistance with temperature an amount equal to the temperature coefficient of 
the copper current posts times the proportional change in the resistance, as may be seen for a special case by a comparison of equations (14) and (13); while obviously any relatively small change in resistance made wholly on the manganin, in such manner as not to perceptibly change the current distribution in the copper, has but little effect on the rate of change of the resistance with temperature.

These conclusions are applicable only in case of a particular type of dissymmetry such as exists in most low-valued precision resistance standards and then only to the extent that the current in the current posts may be considered to be in the direction of their length. They do not apply at all in case of a symmetrical or approximately symmetrical construction, such as is used for some of the heavy-current resistance standards.

\section{EXPERIMENTAL RESULTS}

The first resistance standard selected for adjustment of its temperature coefficient was a Wolff $0.001 \mathrm{ohm}$, constructed approximately 30 years ago. The resistance element consists of two manganin sheets in parallel, each having a width of about 5.5 centimeters and a length of about 13 centimeters. Initially, the potential connections were at the level of the center of the manganin sheets and the rate of increase of resistance with the temperature was 22.5 parts per million per ${ }^{\circ} \mathrm{C}$ at $25 \mathrm{C}$. The intention was to reduce the rate of change of resistance with temperature at $25 \mathrm{C}$ to substantially zero, even though a rough calculation indicated that this would require a greater reduction in the resistance than could be obtained by moving the points of attachment of the potential connections. It was therefore decided to move the potential connections to the bottoms of the current posts, which is equivalent to moving them to points opposite the lower edges of the manganin sheets; redetermine the temperature coefficient, and compare the change with that estimated from the known resistance, dimensions, and assumed values for the conductivity and temperature coefficient of the current posts; then to make further adjustments of the temperature coefficient by reducing the section of the current posts; and finally to readjust the value of resistance without materially changing the temperature coefficient. After having moved the potential connections to the bottoms of the current posts a redetermination of the temperature coefficient showed that it has been reduced substantially the expected amount. However, the resulting change in the resistance was not observed. Then the sections of the current posts were reduced sufficiently to give a total reduction in the resistance equal to that considered necessary to give a zero temperature coefficient at $25 \mathrm{C}$. Then a redetermination of the temperature coefficient showed that while it had been further reduced it was still far from the desired value.

Further reduction of the sections of the current posts resulted in further reductions of the temperature coefficient, but the discrepancy between the change in resistance and expected change in temperature coefficient was so great as to indicate clearly that some unjustifiable assumption had been made. When the temperature coefficient had been reduced to substantially zero, the reduction of the resistance was found to be approximately $2 \frac{1}{2}$ times the estimated amount. In attempting to locate the cause of the discrepancy, it 
was suspected that the temperature coefficient of the copper current posts might be abnormally low, as they are of cast copper. As a direct measurement of the temperature coefficient of the current posts would have presented considerable difficulty, their conductivity was measured and found to be only about 40 percent of normal, so that presumably their temperature coefficient is only about 40 percent of normal. At any rate, the assumption of both the 40 percent normal conductivity and the 40 percent normal temperature coefficient brings observed and expected results into accord. The resistance of the standard was then increased to the desired value by enlarging holes in the manganin sheets as was done in the original adjustment. This decreased the ratio of the copper to the manganin resistance by about 1.5 percent without perceptibly changing the current distribution in the copper. Presumably, therefore, it changed the temperature coefficient about -1.5 percent of about -0.00002 , the estimated contribution of the copper to the temperature coefficient of the standard, or about +0.0000003 , an amount too small to be definitely checked experimentally.

Similar experiments were made on other resistance standards in which the current posts are of drawn copper and therefore presumably have normal values of resistivity and temperature coefficient. The resistance element of each of these standards consists of two or more wires in parallel. Changing the locations of the potential connections on the current posts, or reducing the cross section of the current posts when the potential connections were at the bottoms of the current posts, produced changes in the temperature coefficient equal to 0.0038 times the relative change in the 4 -terminal resistance of the standards, within the experimental error in measuring the temperature coefficients.

\section{RESISTANCE ADJUSTMENTS}

In the construction of resistance standards the ratio of the length to the section of the resistance material cannot be so chosen as to give accurately the desired resistance. Furthermore, after the resistance element has been brazed to the current posts the length cannot conveniently be changed nor the section increased. Therefore, it is customary to make the ratio of the length to the sections such as to give a resistance somewhat low and then bring it up to the desired value by decreasing the section. If, accidentally, the section is reduced too much, or in case the resistance of a standard has increased since its original adjustment, the usual procedure is to attach a shunt of such resistance as will lower the resistance of the standard to the desired value.

As pointed out above, lowering the points of attachment of the potential connections or a reduction of the sections of the current posts below the upper manganin wire or upper edge of the manganin sheet or sheets and above the points of attachment of the potential connections reduces the resistance. This procedure is more convenient and better than the attachment of a shunt.

Should the proposed reduction in the unit of resistance (amounting to about 47 parts in 100,000) be adopted, most of the low-valued precision resistance standards now in use could be made to accord with the new unit by this procedure. Since on the average the resistances 
of these standards are high by about 10 parts in 100,000 the average reduction would amount to about 57 parts in 100,000 .

As most of these standards have copper current posts, an average reduction of 57 parts in 100,000 in the resistance by changes wholly on the current posts would reduce the temperature coefficient on the average about 2 parts in 1,000,000. While in most cases some reduction of the temperature coefficient would be beneficial, such a small reduction would be scarcely significant.

\section{LOAD COEFFICIENTS}

The current in a resistance standard causes the resistance element to assume a temperature higher than that of the surrounding medium, by an amount approximately proportional to the $I^{2} R$ loss in the element, sometimes called the load.

The load coefficient may be defined as the proportional increase in resistance divided by the load. It is generally assumed that, in cases of equal opportunity for the dissipation of the heat, the load coefficients are proportional to the temperature coefficients. While in general this has been found to be true, a considerable number of exceptions have been observed. Usually it has been assumed that these exceptional cases result from strains caused by unequal heating of the resistance element. There is, however, another and possibly more probable reason for many of these exceptions. The temperature rise in the current posts, in general, is small relative to that in the resistance element. Consequently, the load coefficients should be expected to depend mainly on the temperature coefficients of the resistance elements. Therefore, in cases in which the current posts have a material but more or less random effect on the temperature coefficients of resistance standards, it should not be expected that ratios of the load coefficients would be equal to the ratios of the temperature coefficients, even in cases of strictly equal opportunities for the dissipation of heat. Furthermore, a change in the temperature coefficient brought about by changing the location of the potential connections or sections of the current posts should have little or no effect on the load coefficient.

Washington, October 12, 1933. 